\title{
Effect of leaf damage on chlorophyll fluorescence parameters of Eupatorium adenophorum and its genera
}

\author{
Yingzhi Chen ${ }^{1}$, Xingcheng Zhang ${ }^{1}$, Zijun Luo ${ }^{1}$, Gengyun Pan ${ }^{1, *}$, and Ruifang Wang ${ }^{1,2, *}$ \\ ${ }^{1}$ College of Agriculture and Forestry, Puer University, Puer, Yunnan Province 665000, China \\ ${ }^{2}$ Xishuangbanna Tropical Botanical Garden, Chinese Academy of Sciences, Mengla 666303, China
}

\begin{abstract}
Eupatorium adenophorum has a serious impact on agriculture and biodiversity in China. Chlorophyll fluorescence parameters of $E$. adenophorum and its genera were investigated after leaf damage treatments. The results showed that the changes of chlorophyll fluorescence parameters were greatly affected by the treatment methods and species. The changes of chlorophyll fluorescence parameters of $E$. adenophorum and E. lindleyanum after insect feeding were significantly greater than those after simulated insect feeding. Compared with $E$. lindleyanum, E. adenophorum had lower LEF, higher Phi2 and PhiNPQ after damage. Therefore, E. adenophorum had a higher defense ability against insect food damage compared with E. lindleyanum.
\end{abstract}

\section{Introduction}

The weed E. adenophorum Spreng is an invasive alien species native to Central and South America and distributed in many countries and places around the world [1]. The natural enemy escape hypothesis(ERH)states that the reason why alien species can quickly invade a new environment is that they break away from the threat of natural enemies in the native environment and migrate to a new habitat for rapid growth and reproduction[2-3]. Therefore, the use of natural enemies to control the growth of invasive plants has become a research hotspot. Studies have found that E. adenophorum is still facing generalist enemy in the invasive areas. Few studies have been done on the response of E. adenophorum to being grazed by generalist enemy, and whether it is an effective method for biological control of E. adenophorum. The damage caused by feeding by natural enemies can be monitored by chlorophyll fluorescence index.

Chlorophyll fluorescence parameters are a set of values used to describe the photosynthetic mechanism and physiological conditions of plants and reflect the characteristics of plant "internality". They are the internal probes for studying the relationship between plant photosynthesis and environment. Chlorophyll fluorescence parameters can be used to explore the effects of stress on plant physiology and biochemistry. However, there are few studies on the response of chlorophyll fluorescence index of E. adenophorum to the feeding of natural enemies[4].

*Corresponding author: $\underline{704498547 @ \text { qq. } . c o m}$ 


\section{Research materials and methods}

\subsection{Research methods}

Seedling raising and transplanting were completed in the greenhouse of the botanical garden, and humus soil was used as substrate for seedling raising. Transplant when the seedlings are about $10 \mathrm{~cm}$ long, The plants with the same growth were planted in the nutritive bowl. Fertilizer was applied to the plants after the seedlings became viable. were poured into each nutrition bowl.

\subsection{Research treatment and index determination}

Five plants with good growth conditions and basically the same growth were selected, and the two species in each treatment were placed in the same position and marked. In order to avoid errors caused by other insects during the experiment, insecticides (fenpropathrin and carbendazim) should be sprayed on the surface of plant leaves. After spraying, a three-dimensional insectproof net should be used to prevent insecteating. Insects $(H$. armigera as experimental insect) feeding treatment and simulated insect feeding treatment were conducted in the experimental group one to two weeks after pesticide spraying. The experiment was carried out on a sunny day from 11:00 am to 12:00 am. The relatively mature functional leaves (the third leaf from the top to the bottom of the plant) in the feeding treatment and simulation insect feeding treatment were treated, while the control group was not treated. The chlorophyll fluorescence characteristic parameters of the treated leaves were measured with a portable photosynthesizer (Multispeq 2.0) one hour after treatment.

\subsection{Data analysis}

In the data analysis of this experiment, SPSS23 software was used for multivariate analysis and univariate analysis to analyze the significance of the indexes of different plants in the same treatment and the indexes of the same plant in different treatments, and the significance level was selected as $P=0.05$. Use Excel 2010 software for data statistics and chart production.

\section{Experimental results and analysis}

\subsection{Effects of treatment and species on fluorescence parameters}

It can be seen from the table that treatment has significant influence on three parameters of LTD, LEF and Phi2, Phi2 and PhiNPQ were significantly affected by different species.

Table 1. Effects of treatment and species on fluorescence parameters.

\begin{tabular}{cccccc}
\hline factor & & LTD & LEF & Phi2 & PhiNPQ \\
\hline \multirow{2}{*}{ treatment } & $\mathrm{F}$ & 3.673 & 17.755 & 5.000 & 3.373 \\
& $\mathrm{P}$ & 0.041 & 0.000 & 0.015 & 0.051 \\
species & $\mathrm{F}$ & 0.071 & 0.333 & 39.889 & 63.946 \\
& $\mathrm{P}$ & 0.792 & 0.569 & 0.000 & 0.000 \\
\hline
\end{tabular}

$\mathrm{P}$ is the significance of test results $(\mathrm{P}<0.05)$, and $\mathrm{F}$ value is sample variance significance. 


\subsection{Influence of insect feeding and simulated insect feeding on LTD and LEF}

LTD of E. adenophorum and E. lindleyanum treated by insect feeding and simulated insect feeding increased, and the increase of temperature difference between them after insect feeding was greater than that of simulated insect feeding, but there was no significant difference in both.

There was significant difference in LEF between the two species fed by insects and the control. There was no significant difference in LEF between E. adenophorum and E. lindleyanum treated by insect feeding and simulated insect feeding, but there was a significant difference between the two species in the control group.
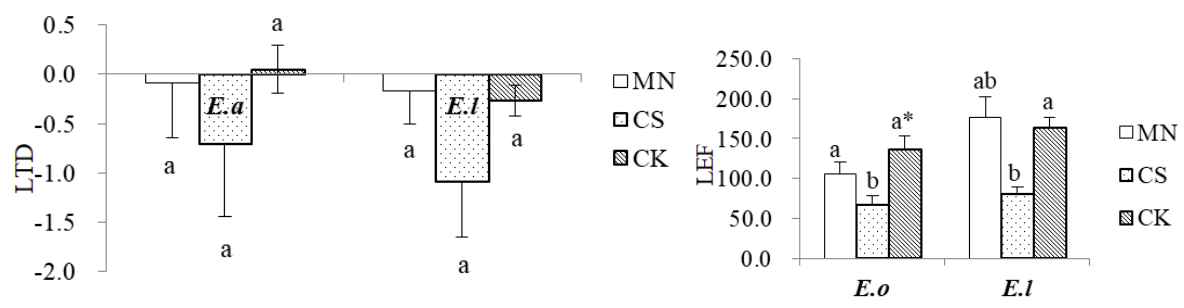

Fig. 1. Impact of insect feeding and simulated insect feeding on LTD and LEF.

In the picture, E. $a$ is Eupatorium adenophorum and $E . l$ is E. lindleyanum. CK means no insect feeding treatment and simulated insect feeding treatment, MN means simulated insect feeding treatment, CS means insect feeding treatment, different lowercase letters mean significant difference between the same parameter or different treatments $(\mathrm{P}<0.05)$, *Indicates significant differences between species, the data in the figure is the mean \pm standard error, the following figure is the same as this figure.

\subsection{Effects of insect feeding and simulated insect feeding on Phi2 and PhiNPQ}

The Phi2 of E. adenophorum after insect feeding was significantly different from that after simulated insect feeding.The Phi 2 of E. adenophorum and E. lindleyanum in simulated and untreated conditions was significantly lower than that in simulated and untreated conditions.

The PhiNPQ of E. adenophorum fed by insects was significantly diffearent from that of simulated insect feeding. There were significant differences in PhiNPQ between $E$. adenophorum and E. lindleyanum species under insect feeding treatment.
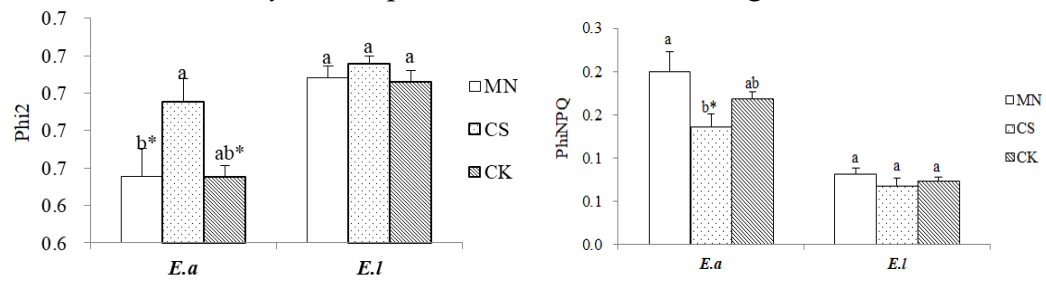

Fig. 2. Impact of insect feeding and simulated insect feeding on Phi2 and PhiNPQ.

\section{Discussion and conclusion}

Decreased in chlorophyll fluorescence may due to the incomplete leaves, and thus LEF and Phi2 decreases. However, in this study, LEF decreased, Phi2 increased after injury of $E$. adenophorum. Therefore, it is speculated that the oral chemicals of insects may induce 
specific signals, leading to significantly higher changes in chlorophyll fluorescence parameters after insect feeding than after simulated insect feeding.

In order to avoid the damage caused by electron accumulation to the membrane system, E. adenophorum may start the electron transport and heat dissipation to consume the excess energy, thus maintaining the high activation of photosystem II . Therefore, $E$. adenophorum had a higher defense ability to damage stress compared with $E$. lindleyanum. PhiNPQ of E. adenophorum is significantly decreased. Which may be due to the initiation of heat dissipation.

In conclusion, it can be seen from the results that E. adenophorum and E. lindleyanum treated by insect feeding and simulated insect feeding all adjust their photosynthetic capacity to adapt to the injury caused by insect feeding. E. adenophorum can compensate effectively when it is fed by the generalist enemy of $H$. armigera, so the damage caused by the invasion of $E$. adenophorum can not be alleviated by the generalist enemy.

This work was supported by the National Natural Science Foundation of China (31660170) a nd Outstanding Young Teacher program (2020GGJS006).

\section{Reference}

1. Zhao Haoyu, Chen Xiaojuan, Liu Jundou, et al. Suggestions on control of several main invasive pests in Sichuan [J]. Sichuan Agriculture and Agricultural Machinery, 2020(02): 51-52.

2. Darwin, the Origin of Species by Means of Natural Selection, 1859.

3. Lan Mingxian, Zhang Mou, Li Jian, et al. Isolation, identification and herbicidal activity of endophytic bacteria from the larva of Musca alba [J]. Acta Agriculturae Jiangxi. 2018, 30(01): 59-64.

4. Liang Junsheng. Photosynthetic characteristics and photosynthetic physiological analysis of Yunnan pine after being attacked by dry bores [D]. Chinese Academy of Forestry Research, 2009. 